\title{
Automated computed tomographic scoring of lung disease in adults with primary ciliary dyskinesia
}

Trieu-Nghi Hoang-Thi ${ }^{1,6}$, Marie-Pierre Revel ${ }^{1}$, Pierre-Régis Burgel ${ }^{2}$, Laurence Bassinet ${ }^{3}$, Isabelle Honoré2 Thong Hua-Huy ${ }^{4}$, Charlotte Martin ${ }^{1}$, Bernard Maitre ${ }^{3}$ and Guillaume Chassagnon ${ }^{1,5^{*}}$ (D)

\begin{abstract}
Background: The present study aimed to develop an automated computed tomography (CT) score based on the $\mathrm{CT}$ quantification of high-attenuating lung structures, in order to provide a quantitative assessment of lung structural abnormalities in patients with Primary Ciliary Dyskinesia (PCD).

Methods: Adult ( $\geq 18$ years) PCD patients who underwent both chest $C T$ and spirometry within a 6-month period were retrospectively included. Commercially available lung segmentation software was used to isolate the lungs from the mediastinum and chest wall and obtain histograms of lung density. CT-density scores were calculated using fixed and adapted thresholds based on various combinations of histogram characteristics, such as mean lung density (MLD), skewness, and standard deviation (SD). Additionally, visual scoring using the Bhalla score was performed by 2 independent radiologists. Correlations between $C T$ scores, forced expiratory volume in $1 \mathrm{~s}\left(\mathrm{FEV}_{1}\right)$ and forced vital capacity (FVC) were evaluated.

Results: Sixty-two adult patients with PCD were included. Of all histogram characteristics, those showing good positive or negative correlations to both $\mathrm{FEV}_{1}$ and FVC were SD $(R=-0.63$ and $-0.67 ; p<0.001)$ and Skewness $(R=0.67$ and $0.67 ; p<0.001)$. Among all evaluated thresholds, the CT-density score based on MLD + $1 S D$ provided the best negative correlation with both $\mathrm{FEV}_{1}(R=-0.68 ; p<0.001)$ and $\mathrm{FVC}(R=-0.71 ; p<0.001)$, close to the correlations of the visual score ( $R=-0.60 ; p<0.001$ for $\mathrm{FEV}_{1}$ and $R=-0.62 ; p<0.001$, for $\mathrm{FVC}$ ).
\end{abstract}

Conclusions: Automated CT scoring of lung structural abnormalities lung in primary ciliary dyskinesia is feasible and may prove useful for evaluation of disease severity in the clinic and in clinical trials.

Keywords: Computed tomography, Primary ciliary dyskinesia, Kartagener syndrome, Bronchiectasis

\section{Background}

Primary ciliary dyskinesia (PCD) is a rare genetic disorder characterized by defective ciliary structure and/or function, leading to inadequate mucociliary clearance and chronic oto-sino-pulmonary disease [1-3]. Organ laterality is also affected in almost half the patients [2]. Defective mucociliary airway clearance leads to recurrent and chronic bacterial infections of the lower respiratory tract, and to bronchiectasis [2].

\footnotetext{
* Correspondence: gchassagnon@gmail.com

${ }^{1}$ Radiology Department, Groupe Hospitalier Cochin-Hôtel Dieu, AP-HP, Université Paris Descartes - Sorbonne Paris Cité, Paris, France

${ }^{5}$ Center for Visual Computing, CentraleSupelec, Gif-sur-Yvette, France Full list of author information is available at the end of the article
}

Computed tomography (CT) is the gold standard method for the diagnosis of bronchiectasis, but its utility for monitoring PCD is not yet established $[4,5]$. Correlations between $\mathrm{CT}$ structural changes and disease severity (lung function) have rarely been studied in PCD, especially in adults [5-13]. However, a large retrospective study recently suggested that a larger disease burden on CT may predict lung function decline in adults with PCD, indicating that CT assessment of lung structural abnormalities might be of value [5].

Most authors who have attempted to quantify bronchial disease in patients with PCD have used visual scoring methods initially designed to assess lung structural changes in patients with cystic fibrosis (CF), but the

(c) The Author(s). 2018 Open Access This article is distributed under the terms of the Creative Commons Attribution 4.0 International License (http://creativecommons.org/licenses/by/4.0/), which permits unrestricted use, distribution, and reproduction in any medium, provided you give appropriate credit to the original author(s) and the source, provide a link to the Creative Commons license, and indicate if changes were made. The Creative Commons Public Domain Dedication waiver (http://creativecommons.org/publicdomain/zero/1.0/) applies to the data made available in this article, unless otherwise stated. 
correlation between these visual scores and forced expiratory volume in $1 \mathrm{~s}\left(\mathrm{FEV}_{1}\right)$ remains controversial in patients with PCD [7-14]. For example, Boon et al. reported a good negative correlation between a visual CT score and $\mathrm{FEV}_{1}(R=-$ 0.63, $P<0.001)$, whereas Cohen-Cymberknoh et al.found no correlation at all $(R=-0.36, P=0.61)[11,12]$.

Although bronchiectasis, bronchial wall thickening, mucus plugging and mosaic perfusion are present in both PCD and CF, their relative predominance differs between the two diseases. Mosaic perfusion and smallairway mucus plugging predominate in PCD, meaning that their respective weight in the overall $\mathrm{CT}$ score should not be the same as in CF $[5,11]$. This may be why some authors failed to find a correlation between visual scores and spirometry in PCD patients. Furthermore, visual scores suffer from several limitations, including the need for dedicated training and subjectivity in the assessment of CT changes [15].

Most lung structural changes in PCD, especially bronchial wall thickening, mucus plugging, consolidations and atelectasis are likely to increase lung attenuation and to modify the density histogram characteristics, which can be extracted from the CT images. On the density histogram, the mode corresponds to the most highly represented attenuation value; and skewness describes the asymmetry of the density curve, which is shifted to the right when there is an increase of lung density or to the left in case of decrease. Additionally, high-attenuating structures can be quantified by using a thresholding approach similar to that used to measure emphysema on CT, except that the latter is based on the quantification of low-attenuating lung areas, with attenuation values below minus 950 Hounsfield units (HU) [16]. The quantification of high-attenuating structural changes in the lungs, also using a thresholding approach has been reported to show good correlation with $\mathrm{FEV}_{1}$ in patients with CF [17].

We postulated that disease severity in PCD might also be assessed by quantifying high-attenuating lung structures and by analysing changes in the lung density distribution. We therefore developed an automated CT scoring method based on histogram characteristic analysis and threshold-based quantification of high-attenuating lung structures in patients with PCD.

\section{Methods}

\section{Patients}

This retrospective study, performed in two accredited PCD reference centres, was approved by the Institutional Review Board of Société Pneumologie de Langue Française. The need for informed consent was waived, in accordance with French rules for retrospective observational studies.
All adult outpatients, with a diagnosis of PCD according to the ERS guidelines [18] were eligible if they had both chest CT exams of the whole thorax performed between November 2009 and July 2016 and spirometric measurements, both performed within a 6-month period. Exclusion criteria were the unavailability of CT images with a slice thickness $\leq 2 \mathrm{~mm}$, reconstructed with a soft kernel, or the administration of iodinated contrast medium during the $\mathrm{CT}$ acquisition.

\section{CT examinations}

All CT examinations had been performed in the supine position at full inspiration, with usual acquisition parameters, allowing obtaining high resolution $\mathrm{CT}$ images of the whole thorax during a single breath hold. Five different 16-to-64 multislice CT devices from two different vendors (Somatom Sensation 16 and Somatom Definition DS, Siemens Healthcare, Erlangen, Germany; Lightspeed plus, Bright Speed 16 and Optima CT 660, GE Healthcare, Milwaukee, Wi) had been used, depending on the site and date of the CT examinations, all performed with equivalent acquisition parameters. The radiation dose resulting from each $\mathrm{CT}$ acquisition was evaluated by collecting the mean dose-length product (DLP) value from the dose reports.

\section{Image analysis}

Pulmonary situs type was identified as solitus, inversus or heterotaxy, based on the relationship between the upper-lobe bronchus and the ipsilateral pulmonary artery, and the morphology of the tracheobronchial tree [19]. CT images were also checked for prior lobectomy.

Lung structural changes were assessed by visual scoring and also by histogram analysis and thresholding of high attenuating lung structures.

\section{Visual CT scoring was performed as follow}

All the images were scored by one thoracic radiologist $(\mathrm{CM})$ using the Bhalla score [20]. Twenty randomly selected examinations were also independently scored by a second radiologist (GC) to assess interobserver repeatability.

\section{Automated CT scoring was performed as follows}

First, the lungs were isolated from the mediastinum and chest wall using a commercially available, automated lung segmentation software (Myrian XP lung software version 1.19.1,Intrasense, Montpellier, France).

This allowed obtaining isolated whole lung volumes, for further density histogram analysis.

We also obtained separate volumes of the upper (right upper lobe and upper part of the left upper lung) and lower lungs (middle lobe, lingula, and lower lobes), after manual contouring of the fissures. This was only done 
for further comparison of the upper and lower lung CT-density scores. Otherwise, the process was fully automated.

The following histogram characteristics were analysed: mean lung density (MLD), mode (the most highly represented attenuation value), standard deviation (SD), kurtosis (sharpness of the density distribution), and skewness (asymmetry of the density distribution).

Lung structural changes having high attenuation values were quantified with a thresholding method, in order to obtain a CT-density score. Several threshold values were tested for their correlation with $\mathrm{FEV}_{1}$ and forced vital capacity (FVC). Three fixed threshold values were tested $(-300,-400$ and $-500 \mathrm{HU})$, as well as eight adapted threshold values taking into account, for each CT examination, individual histogram features, known to be influenced by the inspiratory level [21, 22]. We hypothesized that adapted thresholds based on Mode or MLD or integrating SD might compensate for the changes of density distribution related to the level of inspiration.

The CT-density scores (one for each tested threshold value) were expressed as the proportion of lung showing attenuation values above the selected threshold. For instance, a CT-Density score value of 10 indicated that $10 \%$ of the lung had an attenuation value superior or equal to the threshold on CT.

More details about the whole procedure can be found on a previous work dedicated to automated scoring of CF lung structural changes [17].

\section{Pulmonary function tests}

Forced vital capacity (FVC) and forced expiratory volume in $1 \mathrm{~s}\left(\mathrm{FEV}_{1}\right)$, expressed as the percentage of predicted values, were retrieved from the patients' files. Spirometry was performed as recommended by the American Thoracic Society/European Respiratory Society [23] and predicted values were calculated using the European Community for Steel and Coal reference values [24].

\section{Statistical analysis}

All analyses were done using the ' $R$ ' statistical software package (version 3.2.4, R Foundation, Vienna, Austria). Spearman's correlation coefficient was used to evaluate the correlations between visual scores, histogram characteristics, CT-density scores and spirometry measurements $\left(\mathrm{FEV}_{1}\right.$ and $\left.\mathrm{FVC}\right)$. Spearman $\mathrm{R}$ values were interpreted as follows: $<0.4=$ absent to weak correlation, $0.40-0.59=$ moderate correlation, $0.60-0.79=$ good correlation, $>0.8=$ strong correlation. To evaluate the distribution of high attenuating lung structural changes, the CT-density scores of the upper lung portions (right upper lobe and upper component of the left upper lobe) were compared to those of the lower lung portions (middle lobe, lingula, and lower lobes), using Wilcoxon's paired test. Intraclass correlation coefficients (ICC) and Bland-Altman plots were used to assess interobserver repeatability of the visual scores. Excellent repeatability was assumed when the ICC was 0.8 or more.

\section{Results}

\section{Patients}

Between November 2009 and July 2016, 95 patients with a confirmed diagnosis of PCD were identified, of whom sixty-two patients were included in this study. Among the 33 excluded patients, 24 had no available CT examination, 6 patients had CT scans without soft kernel reconstruction or thin-slice images, and the interval between spirometry and CT exceeded 6 months in the remaining 3 cases (Fig. 1). For the 62 patients who were finally included, PCD diagnosis had been confirmed in 51 by electron microscopy of ciliary ultrastructure. The remaining 11 patients had Kartagener's syndrome with diffuse bronchiectasis and situs inversus on CT imaging, a combination of signs considered to validate PCD diagnosis [13].

Characteristics of the study population are presented in Table 1.

The mean \pm SD age was $39 \pm 15$ years. Mean predicted $\mathrm{FEV}_{1}$ was $67 \pm 20 \%$ and mean predicted FVC was $80 \pm 18 \%$. Their correlation to patients' age was weak $(R=-0.32$, $p=0.012$ for $\left.\mathrm{FEV}_{1}\right)$ and $(R=-0.33, p=0.008$ for FVC).

Regarding other characteristics, 37 patients $(60 \%)$ had the usual arrangement of the pulmonary situs (situs solitus), while 24 (39\%) had situs inversus and 1 (1\%) had left isomerism.

Nineteen patients (31\%) had previously undergone complete or partial lobectomy. The resections concerned the middle lobe in 15 patients (24\%), the left lower lobe in 1 patient (2\%), the middle lobe plus the left lower lobe

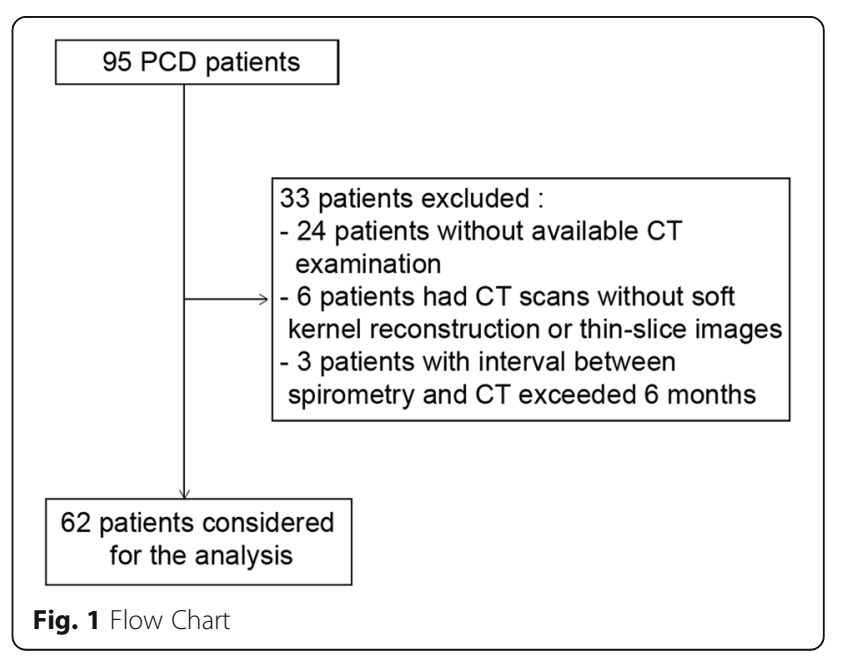


Table 1 Characteristics of the patients $(n=62)$

\begin{tabular}{ll}
\hline Male/Female & $32 / 30$ \\
Age, yr & $39(15)$ \\
Bronchial situs & \\
- solitus & $37(60)$ \\
- inversus & $24(39)$ \\
- left isomerism & $1(1)$ \\
Body mass index, kg/m² & $23.71(4)$ \\
Percentage of predicted FEV 1 & $67(20)$ \\
Percentage of predicted FVC & $80(18)$ \\
$\begin{array}{l}\text { Median interval between spirometry and } \\
\text { CT, months [interquartile range] }\end{array}$ & 0 [0-16] \\
\hline $\begin{array}{l}\text { For quantitative variables, data are mean with standard deviation in parentheses } \\
\text { For qualitative variables, data are numbers of patients, and numbers in parentheses } \\
\text { are percentages } \\
\text { Definition of abbreviations: } C T \text { computed tomography, FEV }{ }_{1} \text { forced expiratory } \\
\text { volume in } 1 \text { s, FVC forced vital capacity }\end{array}$
\end{tabular}

in 2 patients (3\%), and the middle lobe plus the lingula in the remaining patient $(2 \%)$.

\section{CT examinations}

The median interval between CT and spirometry was 0 days [interquartile range: 0-29], 41 of the 62 CT scans being performed on the same day as spirometry. The mean DLP per CT scan was $200.3 \pm 100.4$ mGy.cm.

\section{Visual CT score}

The interobserver repeatability for the visual score was excellent ( ICC $=0.84$ ). Visual CT score, performed for all CT scans by one of the 2 radiologists, showed good correlation with $\mathrm{FEV}_{1}(R=-0.60 ; p<0.001)$ and FVC $(R=-0.62 ; p<0.001)$.

\section{Histogram characteristics}

Two histogram characteristics - kurtosis $(R=0.56$; $p<$ $0.001)$ and skewness $(R=0.60 ; p<0.001)-$ showed a moderate to good correlation to $\mathrm{FEV}_{1}$ (Table 2). The same two characteristics also correlated with FVC ( $R=0.65 ; p<0.001$ and $0.67 ; p<0.001$, respectively). SD showed good negative correlation to both FEV1 $(R=-0.63 ; p<0.001)$ and FVC $(R=-0.67 ; p<0.001)$. Overall the correlations with FVC were slightly stronger than the correlations with $\mathrm{FEV}_{1}$. Examples of variations in histogram shape according to pulmonary function are shown in Fig. 2.

\section{CT-density scores}

All CT-density scores showed moderate to good negative correlations with $\mathrm{FEV}_{1}(R=-0.54$ to $-0.68 ; p<0.001)$ and FVC $(R=-0.62$ to $-0.71 ; p<0.001)$ (Table 2). Overall, CT scores based on fixed thresholds showed weaker negative correlations with $\mathrm{FEV}_{1}(R=-0.51$ to $-0.54 ; p<0.001)$ and FVC $(R=-0.59$ to $-0.62 ; p<0.001)$
Table 2 Correlations between spirometry, histogram characteristics and CT-density scores

\begin{tabular}{|c|c|c|c|c|}
\hline & \multicolumn{2}{|l|}{$\mathrm{FEV}_{1}$} & \multicolumn{2}{|l|}{ FVC } \\
\hline & $\mathrm{R}$ & $P$ value & $\mathrm{R}$ & $P$ value \\
\hline \multicolumn{5}{|c|}{ Histogram characteristics } \\
\hline - MLD & -0.22 & 0.084 & -0.31 & 0.012 \\
\hline - Mode & 0.14 & 0.285 & 0.11 & 0.397 \\
\hline$-S D$ & -0.63 & $<0.001$ & -0.67 & $<0.001$ \\
\hline - Kurtosis & 0.56 & $<0.001$ & 0.65 & $<0.001$ \\
\hline - Skewness & 0.60 & $<0.001$ & 0.67 & $<0.001$ \\
\hline \multicolumn{5}{|c|}{ CT-density score with fixed thresholds } \\
\hline - (-) $400 \mathrm{HU}$ & -0.51 & $<0.001$ & -0.59 & $<0.001$ \\
\hline - (-) $500 \mathrm{HU}$ & -0.53 & $<0.001$ & -0.61 & $<0.001$ \\
\hline - (-) $600 \mathrm{HU}$ & -0.54 & $<0.001$ & -0.61 & $<0.001$ \\
\hline \multicolumn{5}{|c|}{ CT-density score with adapted thresholds } \\
\hline$-M L D+2 S D$ & -0.62 & $<0.001$ & -0.67 & $<0.001$ \\
\hline$-\mathrm{MLD}+1 \mathrm{SD}$ & -0.68 & $<0.001$ & -0.71 & $<0.001$ \\
\hline - Mode + $500 \mathrm{HU}$ & -0.57 & $<0.001$ & -0.64 & $<0.001$ \\
\hline - Mode + $400 \mathrm{HU}$ & -0.60 & $<0.001$ & -0.65 & $<0.001$ \\
\hline - Mode + $300 \mathrm{HU}$ & -0.64 & $<0.001$ & -0.70 & $<0.001$ \\
\hline - Mode + 3 SD & -0.54 & $<0.001$ & -0.62 & $<0.001$ \\
\hline - Mode + 2 SD & -0.65 & $<0.001$ & -0.70 & $<0.001$ \\
\hline - Mode + 1SD & -0.66 & $<0.001$ & -0.68 & $<0.001$ \\
\hline
\end{tabular}

Definition of abbreviations: CT computed tomography, FEV ${ }_{1}$ forced expiratory volume in $1 \mathrm{~s}$, FVC forced vital capacity, HU Hounsfield unit, MLD Mean Lung Density, SD Standard Deviation

than did CT-density scores based on adapted thresholds taking into account histogram characteristics. The strongest correlations were obtained using MLD + 1SD as threshold $\left(R=-0.68 ; p<0.001\right.$ for $\mathrm{FEV}_{1}$ and $R=-0.71$ for FVC; $p<0.001$ ) (Fig. 3). The correlations between CT score and PFTs were in the same range when considering each center separately: $R=-0.66 ; p<0.001$ and -0.68 ; $p<0.001$ for FEV1 and $\mathrm{R}=-0.68 ; p<0.001$ and -0.70 ; $p<0.001$ for FVC. With this threshold value (MLD+ 1SD), the automated score (CT-density score) correlated well with the visual score $(R=0.70, p<0.001)$. Within the Bhalla visual score, the automated CT-density score correlated well with air wall thickening and mucus plugging- related items $(R=0.64$ and 0.61 , respectively; $p<0.001)$ and moderately with bronchiectasis-related items $(R=0.58 ; p<0.001)$ ".

The automated score values were significantly higher in the lower lungs (median: 8.83; interquartile range: 7.61-10.06) than in the upper lungs (median: 6.25; interquartile range: $5.57-6.81)(p<0.001)$.

Results of automated CT scoring in patients with different $\mathrm{FEV}_{1}$ and FVC values are illustrated in Figs. 4 and 5. 


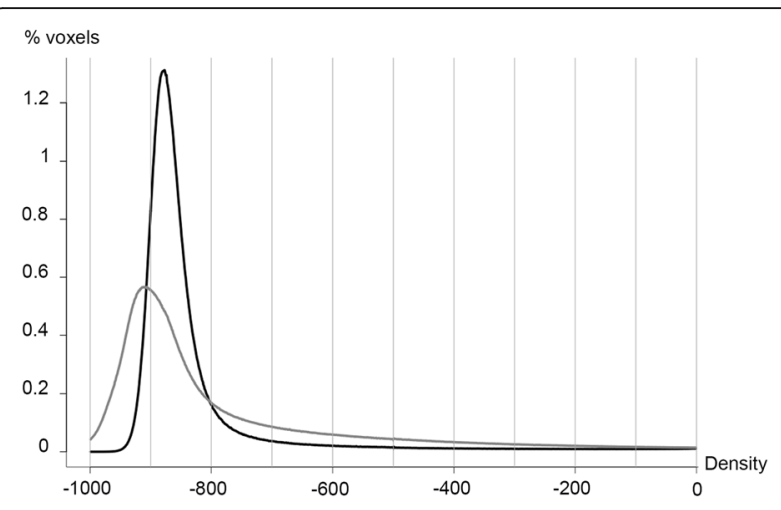

Fig. 2 Variation of histogram characteristics according to lung disease severity. Histogram of lung densities in a patient with mild disease (black line; $\mathrm{FEV}_{1}=81 \% ; \mathrm{FVC}=101 \% ; \mathrm{SD}=110.1$; kurtosis $=31.4$ ). The histogram of lung densities in a patient with severe disease (grey line; $\mathrm{FEV}_{1}=25 \%$; $\left.\mathrm{FVC}=54 \%\right)$ demonstrates higher scattering ( $\left.\mathrm{SD}=213.9\right)$ and flattening (kurtosis $=6.7$ ) of the curve

\section{Discussion}

To the best of our knowledge, the present manuscript describes the first automated CT scoring method designed to quantify lung changes associated with primary ciliary dyskinesia, based on the measurement of high-attenuating structures and considering histogram characteristics on CT. This approach is close to the quantification of emphysema, based on the measurement of low-attenuating lung areas. The method presented here has been previously validated in $\mathrm{CF}$ patients [17].

In the present cohort of 62 adults with PCD, the automated score correlated well with lung function $\left(\mathrm{FEV}_{1}\right.$ and FVC). Moreover, the value of the score was significantly different in the lower and upper portions of the lung, with higher score values in the lower part, consistent with the reported lower lung predominance of bronchial abnormalities in PCD patients [11].

Because the decline of lung function is slower in PCD than in CF, CT is less often performed. However, when performed, lung structural changes on follow-up CT need to be compared to previous images, which is complex in view of the high number of CT images with the modern multidetector technology. Rather than a subjective and time-consuming assessment, automated scoring provides an objective quantitative evaluation.

Regarding the thresholding method, we found that adapted thresholds based on histogram characteristics correlated better than fixed thresholds with the spirometric parameters. Lung attenuation is known to be influenced by parameters such as the level of inspiration, the kilovoltage, and the patient's position in the scan [21, 25, 26]. Expiration and, by extension, a lower level of inspiration, tend to flatten the CT density histogram, resulting in a higher SD and shifting of the curve towards higher density values, which increases Mode and MLD [26]. Instead of evaluating fixed thresholds alone, we postulated that inclusion of histogram characteristics in the threshold definition would compensate for variations not due to disease severity. Among the various thresholds tested here, MLD + 1SD gave the best results. This threshold is readily available, as most commercially available segmentation software programs provide both MLD and $\mathrm{SD}$ values.

One-third of our patients had previously undergone complete or partial lobectomy, even though bronchial abnormalities are not usually restricted to a single lobe in PCD and surgical resection is currently not considered an appropriate treatment for PCD [3]. This proportion is in line with the $41 \%$ prevalence reported by Kennedy et al. In this latter study, where visual CT scoring was performed, a maximal score was arbitrarily affected to the missing lung [14]. In our study, we only applied scoring to the existing lung and found good correlations of the visual score to $\mathrm{FEV}_{1}$ and $\mathrm{FVC}$, in the upper range of previously reported correlations $(0.08$ to -0.63 for $\mathrm{FEV}_{1}$ and -0.38 to -0.60 for $\left.\mathrm{FVC}\right)[8,10,12]$. The correlation of the CT-density score to $\mathrm{FEV}_{1}$ and
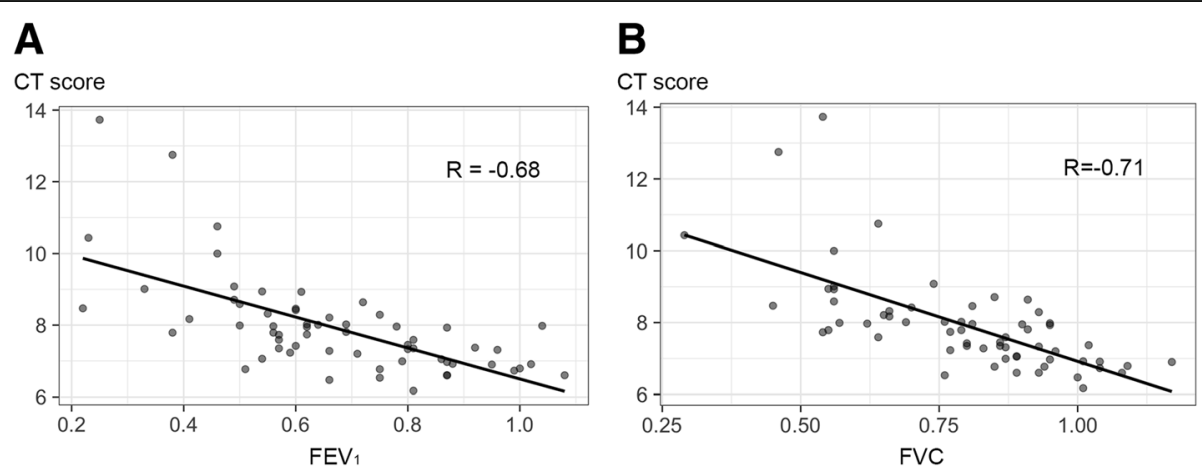

Fig. 3 Relationship between the CT-density score based on MLD + 1SD and lung functional parameters. a Relationship between CT-density score and $\mathrm{FEV}_{1}$. b Relationship between CT-density score and FVC 


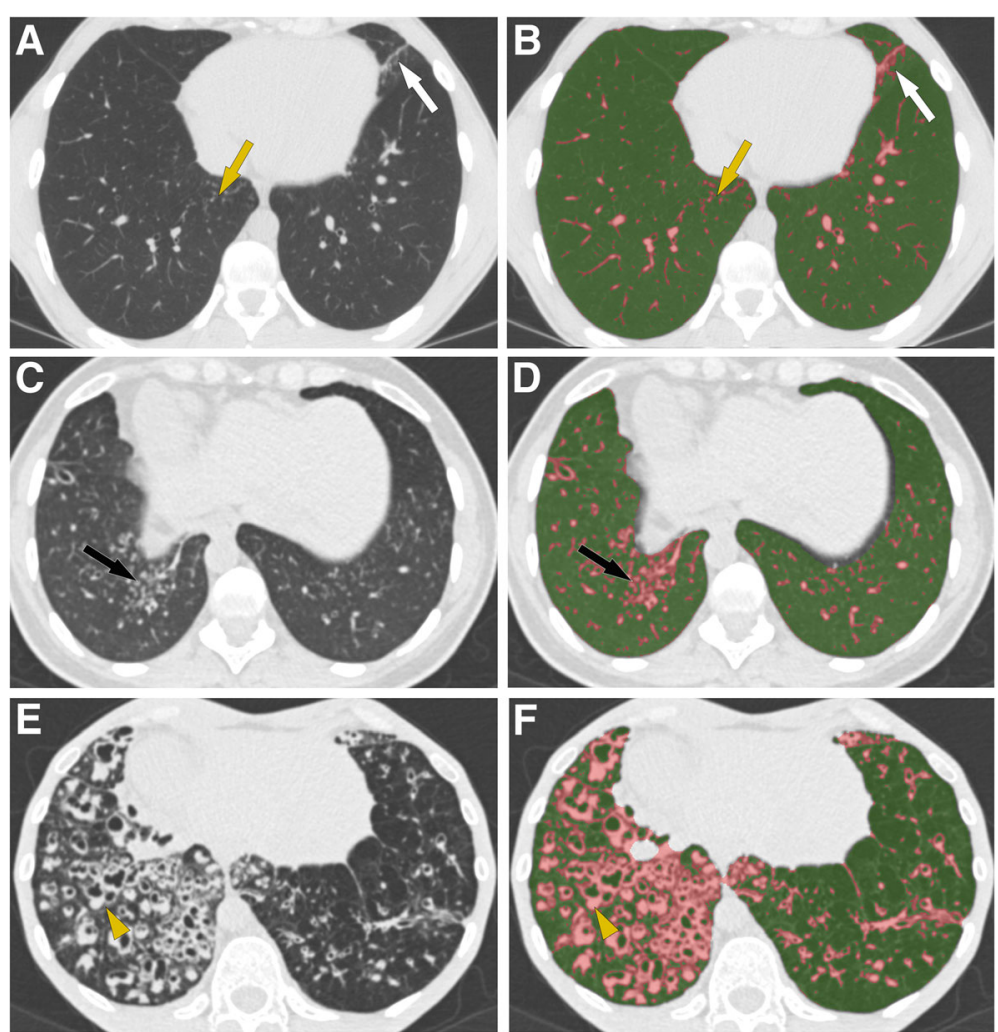

Fig. 4 CT-density scores in patients with increasing disease severity. Areas of high attenuation, using MLD + 1SD as threshold, are tagged in pink. a, b Patient with mild lung involvement ( $\left(\mathrm{FV}_{1}=80 \%, \mathrm{CT}\right.$ score $=6.01$ ): (a) Small areas of tree-in-bud (yellow arrow) and subsegmental atelectasis (white arrow) are seen on the native axial image. b Post-processed image, illustrating that these PCD-related abnormalities appear in pink, along with pulmonary vessels. $\mathbf{c}$, $\mathbf{d}$ Patient with moderate lung involvement ( $\mathrm{FEV}_{1}=60 \%, \mathrm{CT}$ score $=8.37$ ): areas of tree-in-bud (black arrow) seen on the native axial CT image $(\mathbf{c})$ are more extensive and are pink-colored on the post-processed image $(\mathbf{d})(\mathbf{e}, \mathbf{f})$ Patient with severe lung involvement $\left(\mathrm{FEV}_{1}=25 \%\right.$, CT score $\left.=13.72\right)$ : (e) Visually, bronchial abnormalities (yellow arrowhead) predominantly affect the right lung on the native axial CT image. f Post-processed CT image. The CT-density score is 17.59 for the right lung and 10.87 for the left lung

FVC was in the same range, with the advantage of an automated method for the CT-density score.

Because most structural changes (e.g., bronchial wall thickening, mucus plugging) in PCD increase lung density, we based our automated scoring on the identification of high attenuating structures, leaving out empty bronchiectasis and mosaic perfusion. Thus, the automated score mainly quantifies potentially reversible, inflammatory changes such as mucoid impactions, bronchial wall thickening, bronchiolar nodules and consolidations,

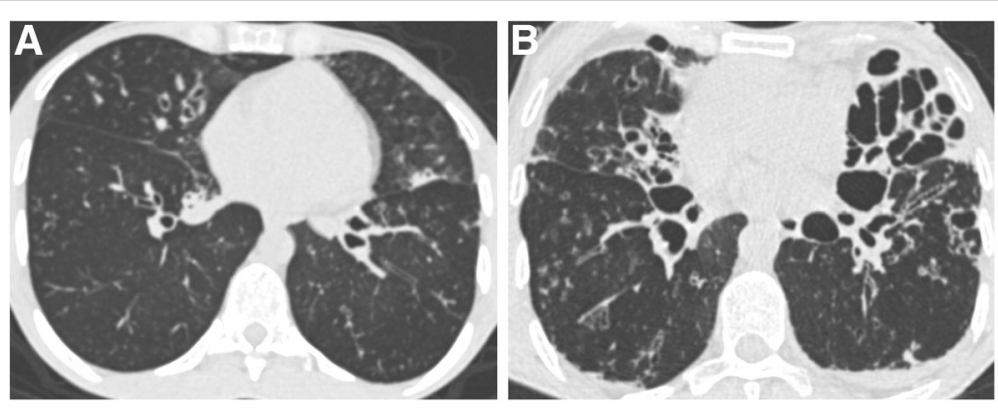

Fig. 5 Different imaging features in patients with similar $\mathrm{FEV}_{1}$ values. a Patient with $\mathrm{FEV}_{1}=38 \%$ predicted and moderate bronchiectasis predominantly affecting the middle lobe. The CT-density score based on MLD + 1SD was 7.79. b Patient with $\mathrm{FEV}_{1}=38 \%$ predicted but much more severe bronchiectasis on visual assessment, especially in the lingula. The CT-density score based on MLD + 1SD was 12.75. These two examples show that $C T$ imaging provides additional information to spirometry, especially regarding regional disease distribution (homogeneous versus heterogeneous), and the severity of bronchiectasis, which correspond to irreversible changes 
whereas it does not consider irreversible changes (e.g., bronchiectasis). Based on these characteristics, we speculate variations in this score may prove useful in identifying worsening of bronchial disease (e.g., during pulmonary exacerbations) or improvement in bronchial patency (e.g., after recovery from a pulmonary exacerbation or due to the beneficial effect of therapy). Due to the unavailability of follow-up chest CT exams for most patients, we were not able to test this hypothesis and future longitudinal studies are needed.

Using our thresholding method, one drawback is that the pulmonary vessels are incorporated into the high-attenuating lung volume, which does not therefore correspond only to the diseased lung. However, differences in pulmonary vessel volume among patients had probably little influence on score variations compared to those due to the bronchial disease, given the good correlation between our score and spirometric parameters. Software capable of pulmonary vessel volume segmentation is being developed [27] and could be used to exclude the pulmonary vessels and improve the performance of the score.

Even though the automated score correlated well with the evaluated functional parameters, we also found that patients with similar $\mathrm{FEV}_{1}$ values could have quite different CT phenotypes. We believe that quantitative assessment of structural changes is of interest in addition to PFT measurements for both cross-sectional evaluation and disease monitoring. For example, disease progression in CF has long relied on assessment of lung function decline whereas CT scan analysis clearly shows that structural abnormalities may appear without significant changes in FEV1 [28]. Thus, CT provides structural information which is complementary to spirometry in patients with $\mathrm{CF}$ and similar findings are likely occurring in patients with PCD. Calculating CT score does not imply additional procedures for the patients since it can be done from standard $\mathrm{CT}$ acquisitions performed as standard of care.

Our study has several limitations. Because this study was retrospective, the CT acquisition parameters were not standardized. This may have influenced the density thresholds. Standardized scanning protocols would probably improve the performance of the developed score. However, the fact that the scoring method can be applied to unstandardized CT examinations makes it suitable for daily clinical use. We did not perform longitudinal evaluation to determine whether changes in the automated CT score correlated with changes in pulmonary function, and whether, as previously suggested, CT-scored disease extent can predict the subsequent decline in pulmonary function. Indeed, our primary objective was to develop and validate an automated CT score by cross-sectional evaluation. Lastly, due to the relative rarity of $\mathrm{PCD}$, it was not possible to split our population into a development and validation cohort. Thus, the developed method should further be validated in an independent cohort of PCD patients.

\section{Conclusion}

In conclusion, automated density-based CT scoring, together with histogram characteristic analysis, is feasible in PCD patients and correlates well with $\mathrm{FEV}_{1}$ and FVC. MLD + 1SD offered the best correlations with both $\mathrm{FEV}_{1}$ and FVC. Quantitative analysis of structural abnormalities on CT scans may prove useful for objectively evaluating lung disease changes in $\mathrm{PCD}$, which may prove useful both in daily clinical use and as an outcome in clinical trials.

\section{Abbreviations}

CF: Cystic fibrosis; CT: Computed tomography; DLP: Dose-length product; $\mathrm{FEV}_{1}$ : Forced expiratory volume in $1 \mathrm{~s}$; HU: Hounsfield units; ICC: Intraclass correlation coefficients; MLD: Mean lung density; PCD: Primary ciliary dyskinesia; SD: Standard deviation

\section{Acknowledgments}

not applicable

\section{Funding}

None

\section{Availability of data and materials}

The datasets used and analysed during the current study are available from the corresponding author on reasonable request.

\section{Authors' contributions}

TTNH, MPR, PRB, BM and GC were involved in conception and design. TTNH, $L B, I H, T H H, C M$ and $G C$ were involved in acquisition and analysis of the data. TTNH, MPR and GC were involved in drafting the manuscript. PRB, $\mathrm{LB}, \mathrm{IH}, \mathrm{THH}, \mathrm{CM}$ and $\mathrm{BM}$ critically revised the work. All authors agreed to be accountable for all aspects of the work.

Ethics approval and consent to participate

Institutional Review Board of Société Pneumologie de Langue Française. The need for informed consent was waived.

Consent for publication

not applicable

\section{Competing interests}

MPR and GC have made a patent application for the image analysis method presented in this article. The other authors declare that they have no competing interests.

\section{Publisher's Note}

Springer Nature remains neutral with regard to jurisdictional claims in published maps and institutional affiliations.

\footnotetext{
Author details

${ }^{1}$ Radiology Department, Groupe Hospitalier Cochin-Hôtel Dieu, AP-HP, Université Paris Descartes - Sorbonne Paris Cité, Paris, France. ${ }^{2}$ Pulmonary Department, Groupe Hospitalier Cochin-Hôtel Dieu, AP-HP, Université Paris Descartes - Sorbonne Paris Cité, Paris, France. ${ }^{3}$ Service de Pneumologie et de Pathologie Professionnelle, DHU A-TVB, Centre Hospitalier Intercommunal de Créteil, Université Paris Est Créteil, Créteil, France. ${ }^{4}$ Physiology Department, Groupe Hospitalier Cochin-Hôtel Dieu, AP-HP, Université Paris Descartes Sorbonne Paris Cité, Paris, France. ${ }^{5}$ Center for Visual Computing, CentraleSupelec, Gif-sur-Yvette, France. ${ }^{6}$ Department Diagnostic Imaging, Vinmec International Hospital - Central Park, Ho Chi Minh City, Vietnam.
} 
Received: 28 August 2018 Accepted: 4 December 2018

Published online: 18 December 2018

\section{References}

1. Afzelius BA. A human syndrome caused by immotile cilia. Science. 1976;193: 317-9.

2. Meeks M, Bush A. Primary ciliary dyskinesia (PCD). Pediatr Pulmonol. 2000; 29:307-16

3. Honoré I, Burgel P-R. Primary ciliary dyskinesia in adults. Rev Mal Respir. 2016;33:165-89.

4. Barbato A, Frischer T, Kuehni CE, Snijders D, Azevedo I, Baktai G, et al. Primany ciliary dyskinesia: a consensus statement on diagnostic and treatment approaches in children. Eur Respir J. 2009:34:1264-76.

5. Shah A, Shoemark A, MacNeill SJ, Bhaludin B, Rogers A, Bilton D, et al. A longitudinal study characterising a large adult primary ciliary dyskinesia population. Eur Respir J. 2016;48:441-50

6. Kennedy MP, Noone PG, Leigh MW, Zariwala MA, Minnix SL, Knowles MR, et al. High-resolution CT of patients with primary ciliary dyskinesia. AJR Am J Roentgenol. 2007;188:1232-8.

7. Santamaria F, Montella S, Tiddens HAWM, Guidi G, Casotti V, Maglione M, et al. Structural and functional lung disease in primary ciliary dyskinesia. Chest. 2008:134:351-7.

8. Maglione M, Bush A, Montella S, Mollica C, Manna A, Esposito A, et al. Progression of lung disease in primary ciliary dyskinesia: is spirometry less accurate than CT? Pediatr Pulmonol. 2012;47:498-504.

9. Magnin ML, Cros P, Beydon N, Mahloul M, Tamalet A, Escudier E, et al. Longitudinal lung function and structural changes in children with primary ciliary dyskinesia. Pediatr Pulmonol. 2012;47:816-25.

10. Pifferi M, Bush A, Pioggia G, Caramella D, Tartarisco G, Di Cicco M, et al. Evaluation of pulmonary disease using static lung volumes in primary ciliary dyskinesia. Thorax. 2012;67:993-9.

11. Cohen-Cymberknoh M, Simanovsky N, Hiller N, Gileles Hillel A, Shoseyov D, Kerem E. Differences in disease expression between primary ciliary dyskinesia and cystic fibrosis with and without pancreatic insufficiency. Chest. 2014;145: 738-44.

12. Boon M, Vermeulen FL, Gysemans W, Proesmans M, Jorissen M, De Boeck K. Lung structure-function correlation in patients with primary ciliary dyskinesia. Thorax. 2015;70:339-45.

13. Frija-Masson J, Bassinet $L$, Honoré I, Dufeu N, Housset B, Coste A, et al. Clinical characteristics, functional respiratory decline and follow-up in adult patients with primary ciliary dyskinesia. Thorax. 2017:72:154-60.

14. Kennedy MP, Omran H, Leigh MW, Dell S, Morgan L, Molina PL, et al. Congenital heart disease and other heterotaxic defects in a large cohort of patients with primary ciliary dyskinesia. Circulation. 2007;115: 2814-21.

15. Calder AD, Bush A, Brody AS, Owens CM. Scoring of chest $C T$ in children with cystic fibrosis: state of the art. Pediatr Radiol. 2014;44:1496-506

16. Gevenois PA, de Maertelaer V, De Vuyst P, Zanen J, Yernault JC. Comparison of computed density and macroscopic morphometry in pulmonary emphysema. Am J Respir Crit Care Med. 1995:152:653-7.

17. Chassagnon G, Martin C, Burgel P-R, Hubert D, Fajac I, Paragios N, et al. An automated computed tomography score for the cystic fibrosis lung. Eur Radiol. 2018.

18. Lucas JS, Barbato A, Collins SA, Goutaki M, Behan L, Caudri D, et al. European Respiratory Society guidelines for the diagnosis of primary ciliary dyskinesia. Eur Respir J. 2017:49:1601090.

19. Chassagnon G, Morel B, Carpentier E, Ducou Le Pointe H, Sirinelli D. Tracheobronchial branching abnormalities: lobe-based classification scheme. Radiographics. 2016;36:358-73.

20. Bhalla M, Turcios N, Aponte V, Jenkins M, Leitman BS, McCauley DI, et al. Cystic fibrosis: scoring system with thin-section CT. Radiology. 1991;179:783-8.

21. Goris ML, Zhu HJ, Blankenberg F, Chan F, Robinson TE. An automated approach to quantitative air trapping measurements in mild cystic fibrosis. Chest. 2003;123:1655-63.

22. Goris ML, Zhu HJ. Robinson TE. A critical discussion of computer analysis in medical imaging. Proc Am Thorac Soc. 2007:4:347-9.

23. Miller MR, Hankinson J, Brusasco V, Burgos F, Casaburi R, Coates A, et al. Standardisation of spirometry. Eur Respir J. 2005;26:319-38.

24. Quanjer PH, Tammeling GJ, Cotes JE, Pedersen OF, Peslin R, Yernault JC. Lung volumes and forced ventilatory flows. Report working party standardization of lung function tests, European Community for steel and coal. Official statement of the European Respiratory Society. Eur Respir J Suppl. 1993;16:5-40.

25. Robinson PJ, Kreel L. Pulmonary tissue attenuation with computed tomography: comparison of inspiration and expiration scans. J Comput Assist Tomogr. 1979;3:740-8.

26. Levi C, Gray JE, McCullough EC, Hattery RR. The unreliability of $C T$ numbers as absolute values. AJR Am J Roentgenol. 1982;139:443-7.

27. Rudyanto RD, Kerkstra S, van Rikxoort EM, Fetita C, Brillet P-Y, Lefevre C, et al. Comparing algorithms for automated vessel segmentation in computed tomography scans of the lung: the VESSEL12 study. Med Image Anal. 2014;18: 1217-32.

28. Loeve M, Krestin GP, Rosenfeld M, de Bruijne M, Stick SM, Tiddens HA. Chest computed tomography: a validated surrogate endpoint of cystic fibrosis lung disease? Eur Respir J. 2013;42:844-57.
Ready to submit your research? Choose BMC and benefit from:

- fast, convenient online submission

- thorough peer review by experienced researchers in your field

- rapid publication on acceptance

- support for research data, including large and complex data types

- gold Open Access which fosters wider collaboration and increased citations

- maximum visibility for your research: over $100 \mathrm{M}$ website views per year

At BMC, research is always in progress.

Learn more biomedcentral.com/submissions 